\title{
Key aspects on cancer immunology
}

\begin{abstract}
Although the obvious achievements in treatment and diagnostics are available, oncology diseases remain a major healthcare problem worldwide. The incidence of cancer has significantly increased globally due to the increasing exposure of risk factors and average life expectancy. The specific response against tumours is developed and realized by the immuno competent organs. However, activated immuno competent cells are frequently failed in their mission to reject the tumor, which leads to cancer progression and metastasis. Such failure of immune system is linked with the phenomenon of cancer recurrence, which is based on widespread of highly metastatic tumor cells with low immunogenicity. Thus, the tumor cells acquire the ability to avoid the monitoring of immune system and become resistant to anti-cancer drugs. The immune response during cancer as well as immune escape is the complex of different processes those should be further investigated and determined. The article aims to present the main immunology mechanisms specific to cancer.
\end{abstract}

Keywords: cancer specific genes, immune escape, PD-1/PD-L1 signalling, polymorphism, Treg
Research Article

Volume 5 Issue I - 2018

\section{Ekaterina KIdiashvili, Lana Bokuchava, Sophio Bojgua, Dodo Agladze \\ Molecular Medicine and Laboratory Technologies, New Vision} University, Georgia

\section{Correspondence: Ekaterina KIdiashvili, Molecular Medicine and Laboratory Technologies, New Vision University, Georgia, Tel +995599957328, Email ekldiashvili@yahoo.com}

Received: January 23, 2018 | Published: March 01, 2018

\section{HLA class I complex and tumor}

The function and role of immune system to develop a T-lymphocytes based specific immune response against tumor is well known and described. However, the tumor cannot be frequently rejected by activated tumor-specific immuno competent cells. This functional downfall of immune response is the background mechanism of tumor progression and metastasis. This phenomenon is based on widespread of highly metastatic tumor cells with low immunogenicity. The molecular background of antitumor immune response and cancer immunotherapy is the recognition by cytotoxic $\mathrm{T}$ lymphocytes (cytotoxic T-cells, CTLs) the heavy chain/beta2-microglobulin $(\beta 2 \mathrm{~m}) /$ tumor peptide, which belongs to human leukocyte antigen (HLA) class I complex. ${ }^{1,2}$ Therefore, the cancer immune escape and metastasis development are associated with altered and defective expression of HLA class I molecules in tumor tissue. ${ }^{2,3}$ The stimulation by cytokines or immunotherapy might be helpful for recovery of some HLA alterations, so called "soft" lesions. We suppose, that the escape from immune recognition is linked with tumor cells specific "hard" lesions - inevitable structural defects. ${ }^{4}$ The low response to immunotherapy and progressive metastases in melanoma patients are described for HLA-negative tumor cellular variants with irreversible defects. ${ }^{5}$ Therefore, the determination of the tumor specific HLA class I defect (regulatory or structural) is highly important for selection of an appropriate immunotherapy protocol. Furthermore, melanoma specific tumor HLA class I antigen expression is determined as "immunological constant of rejection". The tumor HLA class I antigen expression in melanoma correlates with tumor rejection mediated by CTLs, allograft rejection, graft-versus-host disease or development of an autoimmune disease. ${ }^{6}$ It has been revealed, that $\beta 2 \mathrm{~m}$ deficiencies are underlying the mechanisms of immune escape in melanoma and other types of cancer. ${ }^{7}$ Two genetic events targeted on $\beta 2 \mathrm{~m}$ gene are underlying the $\beta 2 \mathrm{~m}$ loss in cancer cell; these mechanisms are mutation of one copy of target gene and loss of its copy. ${ }^{8}$ The chronological order of these events should be further investigated. $\beta 2 \mathrm{~m}$ loss in cancer cells correlates with tumor tissue specific HLA class I abnormalities and as a consequence with a worse clinical outcome. HLA alterations can be seen in neoplastic tissues and cell cultures developed from tumor tissues of clinical samples. ${ }^{7,9}$ Unfortunately, the $\beta 2 \mathrm{~m}$ genetic changes and alterations developed during and specific to metastasis have not been investigated till now. Only several research projects were focusing on correlation between HLA classes I loss and immune escape. In these experiments neoplastic tissue samples and cell cultures developed from the mentioned tissue samples were investigated. The experimental data links the HLA class I genetically altered expression with metastasis development. ${ }^{7,10}$ We hypothesize, that the correlation between immune escape of HLA I negative tumor tissues and $\beta 2 \mathrm{~m}$ loss exists. Probably this is an early genetical event of cancer progression.

\section{CTLs programmed death I receptor and cancer cell programmed death ligand}

The escape of tumor from immunological reactions as well as acquired resistance to anti-cancer drugs is the unique and specific features of cancer cells. They are the key barriers to the successful management of oncology disease. The main mechanism of cancer escape from immunological reactions is based on interaction between Programmed Death 1 (PD-1) receptor of CTLs and Programmed Death Ligand 1 (PD-L1) of cancer cell. ${ }^{11}$ The PD-1/PD-L1 interaction is important event as a part of "immune checkpoint regulators". This interaction is important for development of self-tolerance; this is the limiting factor of immune response duration and strength. The mechanism of this interaction is based on inhibition of adaptive $T$ cell responses. ${ }^{12}$ The tumor cells realized immune regulation mechanism of PD-1/PD-L1 aims anti-cancer adaptive responses suppression. In particular, the activation of PD-1/PD-L1 mechanism correlates with anti-tumor adaptive responses suppression, those are based on induction of CTL anergy, exhaustion, apoptosis and decreased cytokine production. ${ }^{11,13}$ Furthermore, PD-1 and PD-L1 mechanism 
is linked with obvious resistance of cancer cell to pro-apoptotic signals. These signals are delivered by cytotoxic immune effectors, staurosporin, as well as Fas ligation. ${ }^{14}$ The precise cellular mechanism of this phenomenon should be further determined and investigated. There is the difference in cancer cells specific expression of PDL1. PD-L1 expression is stimulated by local factors and molecules like interferon gamma (IFN $\gamma)^{13,15}$ Therefore, PD-L1 is a valuable prognostic marker; PD-L1 expression correlates with the grade of tumor infiltration by lymphocytes (TILs), ${ }^{16}$ high histological grade of tumor $^{17}$ and worse clinical outcome ${ }^{18}$ is already determined. Nowadays research activities are focused on PD-1/PD-L1 signalling through the application of humanized monoclonal antibodies (e.g., Nivolumab). The obtained results confirmed obvious clinical responses in advanced cases of skin cancer (melanoma), lung cancer (non-small cell lung cancer), kidney cancer (renal cell carcinoma) and etc. Furthermore, recent data suggest that PD-1/PD-L1 mechanism may be a background of cancer cell survival mechanism. It has been discovered that PD-1/PD-L1 based mechanism may be a background of resistance to radiotherapy and anti-CTLA-4 antibody based immunotherapy. ${ }^{19}$ It may be estimated, that response to PD-1/PD-L1 blockade therapy is associated with tumor PD-L1 protein levels. ${ }^{20}$

Taking into account that PD-L1 expression protects of tumor cells from influence of pro-apoptotic agents, ${ }^{14}$ and that the PD-1/PD-L1 mechanism correlates with severe clinical outcomes ${ }^{18}$ we hypothesize that this mechanism is linked with development and acquiring of resistance to conventional chemotherapeutic drugs. We propose that inhibition of PD-1/PD-L1 mechanism by applying of PD-1 targeted therapy will enhance the efficacy of conventional chemotherapy. The biomarkers for predicting the efficacy of the PD-1 blockade cancer therapy should be mentioned especially. Potential predictive biomarkers for anti-tumor responses with PD-1 inhibitors can be found among tumor cell-related factors and host immunological factors. The frequency of cancer cells specific genetic mutations due to microsatellite instability (MSI) combined with DNA mismatch repair deficiency (MMRd) has been recently reported as a candidate biomarker. ${ }^{21-24}$ The mutated new superficial antigens of cancer cells are recognized by $\mathrm{T}$ and $\mathrm{B}$ lymphocytes as foreign antigens directly or through the antigen presenting cell (APC) system. Cancer cells exposed to IFN- $\gamma$ released from activated $\mathrm{T}$ cells express PD-L1; they are establishing an acquired immune resistance. ${ }^{24}$ In such cases PD-1 signal inhibitors are effective. Experimentally were examined candidate biomarkers such as the PD-L1 level on tumor cells and the frequency of tumor-infiltrating lymphocytes, ${ }^{25,26}$ the levels of IFN- $\gamma$-related genes in tumor cells,,${ }^{27,28}$ the frequency of mutations in tumor cells, ${ }^{21-24}$ and the diversity of TCRs in tumor antigen-specific $\mathrm{T}$ cells..$^{24,29,30}$ It has been revealed that these candidates do not always correspond to a high response according to cancer type. For example, clinical trials of PD-1 inhibitors for squamous-cell lung cancer and ovarian cancer showed no correlation between clinical effect and PD-L1 expression on tumor tissues. ${ }^{31}$ The high mutational loads and genes related to T-cell checkpoints, such as CD8A/B, PD-L1, LAG3, and IFN- $\gamma$, have been revealed; but in tumor tissues they were not associated with responsiveness in breast cancer patients. ${ }^{32}$ The breast cancer susceptibility gene (BRCA) 2 mutation status is associated with responsiveness to PD-1 mAb therapy, ${ }^{32}$ while no correlation was found between BRCA2 and avelumab's clinical effect on ovarian cancer. ${ }^{31}$ It is urgent to validate current candidates and to discover new biomarkers for clinical response to PD-1 signal inhibitors.

\section{Cell signaling during cancer}

It is well known, that signaling systems of the cell are important for tissue/organ development and repair, entering and progression of the cell through cell cycle, cellular migration and survival within microenvironments. ${ }^{33}$ These signaling systems function in a tissue in cell-type specific manner for the aim to realize cell cycle, antiapoptotic and cellular movement mechanisms. ${ }^{34,35}$ It has been revealed that the most oncogenic mutations alter the functionality of the cellular signaling systems ${ }^{36}$ regulation of mitosis, apoptosis and stipulate cellular movement. ${ }^{37}$ The most important signaling events and their role in cancer are highlighted below. Receptor Tyrosine Kinases (RTKs) are growth factor receptors, those ensure the ability of the normal cell to survive and proliferate. ${ }^{38}$ As a result of ligand binding occurs oligomerization of RTKs and phosphorylation of tyrosine residues within the receptor tails or on surrogate proteins (such as IRS, Gab, and FRS).$^{35}$ This is the key event of RTKs mediated intracellular events initiation. Oncogenic mutations of RTKs are deregulating activation of receptor tyrosine kinases or their downstream signaling components. For example, HER 2 is amplified in about $30 \%$ of breast cancers and $4 \%$ of non small cell lung cancer (NSCLC) cases. HER 1 is active in glioblastomas and in $10 \%$ of NSCLC patients. ${ }^{38,39}$ Activating mutations of KIT and PDGFR have been revealed in gastrointestinal sarcomas. ${ }^{40}$ Furthermore, PDGF and its receptor are overproduced in glioblastomas. ${ }^{38} \mathrm{KRAS}$ and BRAF activating mutations are frequent in melanoma, pancreatic adenocarcinoma, colorectal carcinoma, and NSCLC cases. ${ }^{41,42}$ Loss of hetero zygosity of PTEN is one of the most common genetic alterations observed in tumor tissues. ${ }^{43,44}$ Activating mutations of:

a. JAK2 kinase in myelo proliferative neoplasms,

b. KIT mutations in mucosal melanoma, and

c. FGFR2 mutations in endometrial carcinoma have been revealed recently. ${ }^{45}$

Cell Adhesion Receptors are mediating the response of normal cells to mitogen when they are not inhibited by cadherin-mediated contact inhibition and are attached to extracellular matrix through integrins. Cancer cells are not responding to these control mechanisms. ${ }^{46,47}$ It is well known, that the most integrins activate Focal Adhesion Kinase (FAK) and Src family kinases, another integrins interact and activate RTKs. It has been hypothesized, that deregulated integrin signaling contributes to tumor formation. Amplification of FAK in breast cancer and over activation of Src family kinases in colorectal cancer has been revealed. ${ }^{48,49}$ The consequence of contact inhibition is the formation of cell junctions. This ensures the immobilization of $\beta$-catenin, prohibiting of its participation in Wnt signaling ${ }^{50}$ and attenuate the activation of receptor tyrosine kinases. ${ }^{51}$ It has been recently revealed, that loss of contact inhibition contributes to the invasive growth of cancer cells. Inactivation of E-cadherin has been documented in diffuse gastric and lobular breast cancers. ${ }^{52}$ It has been revealed, that JAG1 and SNA1 up regulation in cancer cells is increased by platelets. ${ }^{53} \mathrm{JAG} 1$ is encoding a ligand for the Notch receptor, which is inducing SNAI2 expression. SNAI2 together with SNAI1 belong to a complex of transcription factors those are responsible for epithelialmesenchymal transition and E-cadherin inhibition. Accordingly with our hypothesis platelet adhesion to cancer cells stipulates the development of epithelial-mesenchymal transition (EMT) phenotype. We suppose that this event is the functional and molecular background of cancer cell migration, extra vacation and metastasis development. 


\section{Immune response during cancer}

The interactions between the immune system and the tumor are complex events those will result in successful tumor eradication or immune evasion by the tumor. ${ }^{54}$ Tumor development and survival depends on interaction of cancer and normal cells, cancer cells and local microenvironment, as well as cancer cells and immune system. In general, $\mathrm{T}$ lymphocytes (CD8+ cytotoxic T cells - CTLs and CD4+ helper $\mathrm{T}$ cells) curb tumor development through production of interferon (IFN)- $\gamma$ and cyto toxins, ${ }^{55}$ but other factors like chronic infection can inhibit these effects and promote tumor development. ${ }^{56,57}$ It has been revealed, that chronic inflammation plays a critical role in the development of cervical, prostate, colon and pancreatic cancers. ${ }^{58}$ Therefore, the chronic infection induced promotion of tumor growth and angiogenesis occurs at failure of acute immune response. The link between autoimmune disease and development of lymphoma has been reported too. ${ }^{58}$

The immune system is maintaining the equilibrium between immune recognition and tumor development. The outcome may be promotion or suppression of tumor growth. By the analysis of the existed research data the concept of "immuno editing" has been developed. ${ }^{59-61}$ It is helpful for explanation why in some cases tumours are dormant for years before re-emerging, and why other cases of tumours grow under the conditions of a fully functional immune system. ${ }^{62}$ In the process of cancer immuno editing, the presenting tumor antigens most immunologically vulnerable cancer cells are eliminated because of their recognition and destroyment by the immune system. ${ }^{63}$ As a result of genetic instability, permanent tumor cell division can generate malignant cells with reduced immunogenicity and ability to evade immune elimination. The process of formation of new tumor cell variants balanced by the elimination is duplicating "equilibrium", during which the cancer cells continue division and accumulation of accidental mutational change or as response to chronic inflammation. In such case the balance between immune control and tumor growth is maintained, giving the appearance of tumor dormancy. ${ }^{64}$ However, these processes are the background of impairment of capacity of the immune system by tumours. The plausible development is the tumor eradication due to development of immune suppressive effects or discontinuation of expression of target antigens. This is the moment of tumor escape and formation of obvious clinical cancer. Some conditions may be considered as truly dormant cases of tumor. This might be achieved through induction of "senescence". In such cases tumours remain dormant permanently as a result of irreversible replicative senescence. ${ }^{65}$ It has been shown that concrete genes are related to immune response. ${ }^{66}$ their expression rates are low in primary colorectal carcinomas with ability of later metastasis development. It is plausible, that the immune-related genes reduced expression alters activation of CD4 T-cells mainly through the MHC class II pathway. Platelets and circulating tumor cells (CTCs) interactions are important for haematogenous metastasis development. ${ }^{53}$

\section{FOXP3 gene and metastasis development}

Although the genes critical for the concrete cancer disease development are identified, the genes responsible for metastasis development and as a consequence for cancer recurrence aren't identified till now. One of candidates is FOXP3. FOXP3 (Forkhead box protein P3) is important as regulatory T cells (Tregs) key transcription factor. It is important for suppression of Tregs immune functions. ${ }^{67}$ The recent research confirmed the expression of FOXP3 in tumor cells. ${ }^{68-70}$ This gene expression in tumor is linked with the mechanism of cancer immune escape. ${ }^{71}$ It has been revealed, that FOXP3 is expressed in cancer cells and Tregs. Cancer cells can induce transformation of $\mathrm{T}$ cells into Tregs and by this realize the immune escape mechanism. ${ }^{72}$ The availability of Tregs in neoplastic samples probably is making influence on tumor microenvironment. Furthermore, the availability of Tregs in tumor samples is linked with poor prognosis and worse clinical outcomes. ${ }^{73-75}$ The molecular features of FOXP3 should be noted too. FOXP3 is located at the short arm of X chromosome. The conserved non-coding sequences in intronic regions of FOXP3 bind with transcriptional factors; the expression regulation of the gene is complex. ${ }^{76}$ Polymorphisms of above mentioned intronic regions of FOXP3 could alter not only binding of transcriptional factors, but also expression of FOXP3 and function of Tregs by themselves. ${ }^{77}$ The polymorphism rs2232365 (A to G) of FOXP3 regulatory region correlates with immunologic diseases. These data are emphasizing the importance of FOXP3 gene for Tregs function regulation. The role of FOXP3 and its polymorphism rs2232365 (A to G) has not been investigated in cancer till now. Taking into account all above mentioned it can be hypothesized, that FOXP3 gene and its rs 2232365 $\mathrm{A} / \mathrm{G}$ polymorphism are associated with cancer. Furthermore, it is plausible that FOXP3 rs2232365 A/G polymorphism contributes to anesthesia drug adverse effect and the increased risk of cancer recurrence.

\section{Cellular interactions in cancer}

Tumours are composed by the different cellular types. Among those can be distinguished cancer cells as well as cancer-associated fibroblasts (CAFs), specialized mesenchymal cell types specific to concrete tissue environment, immune competent cells, endothelial cells and pericytes. The extracellular matrix (ECM) composed by structural (collagen and elastin) and specialized (fibrillin, fibronectin and elastin) proteins and proteoglycans is the essential component of the tumor too. ${ }^{78}$ Research data indicate, that the development of cancer strongly depends on tumor microenvironment and cellular interactions specific to the tumor. Furthermore, the importance of the Stephen Paget's 'seed and soil' theory (1889) cannot be underestimated. Accordingly with this theory metastases of a concrete cancer ('the seed') often localizes to specific sites ('the soil', such tropism is due to the similarity of the environments of the original and secondary tumour sites. ${ }^{79}$ This theory has been confirmed experimentally. It has been revealed, that the tumour microenvironment (TME) is the mentioned 'soil' ${ }^{80-83} \mathrm{In}$ tumor development and metastasis TME determines the underlying processes. The TME is important for deregulation of ECM, activation of proliferative signalling, inhibition of suppressors and apoptosis, activation of invasion and metastasis, and etc. The factors produced and secreted by primary tumours are altering the microenvironment of distant organs and by this making them suitable and attractive target for colonization by metastatic cancer cells. The non-malignant cells of tumor stroma produce the specific microenvironment which can modify the neoplastic properties of the malignant cells. ${ }^{84}$ The importance of TME, is supported by the concept that cancer cells aren't manifesting the disease by themselves, they alter and influence the change of recruited normal cell types. ${ }^{85}$ Thus, the niche, or local microenvironment, of a cancer cell is extremely important for tumour progression. In 2012 has been proposed ${ }^{85}$ distinguishing of the following cellular types in TME stromal component: 
a. Angiogenic vascular cells,

b. Infiltrating immune cells and

c. Cancer-associated fibroblastic cells.

These different cellular populations interact with each other and contribute to cancer cells growth, local invasion and metastasis. Fibroblasts are the predominant cells of the stroma. It has been revealed, that genetic and epigenetic changes in stromal fibroblasts may change the expression of genes encoding growth factors and cytokines. ${ }^{86,87}$ Fibroblasts are producing ECM molecules (i.e., fibronectin and tenascin), those influence the processes of cell adhesion and proliferation.$^{84}$ Myeloid cells, especially macrophages, are important for metastatic cascade development..$^{88}$ The macrophages are diploid; these cells are characterized with low mutation rates. Therefore, macrophages cannot easily develop drug resistance and may be used as effective targets for anti-cancer therapy. It should be noted, that modern anti-cancer therapies are targeting all macrophages through inhibition of CFS1R or CCR2 for example, which is the limitation factor of macrophage targeted therapies. The focused investigation of cellular interactions, their molecular mechanisms and specific microenvironment of tumor is required and essential for development of effective and precise anti-cancer therapy. The discussion of cellular interactions during cancer will not be complete without overview of epithelial-mesenchymal transition. It is a developmental process during which epithelial cells transdifferentiate into mesenchymal cells and migrate to other regions of the embryo to form new cellular types. EMT is silent in normal, healthy tissues. However, its reactivation occurs in cancer ${ }^{89}$ Epithelial cells undergoing EMT lose epithelial characteristics. This means that cells aren't forming tight and adherens junctions any more, they lose apico-basal cellular polarity, and the cytoskeleton of such cells is reorganized. Cell is still able to migrate at new location, it became invasive. The highly invasive phenotype in human carcinomas is associated with the loss of E-cadherin expression..$^{90}$ The regulation of E-cadherin is among the topics of high research interest. The transcription factors those are inducing EMT are Snail1/2, ZEB1/2, and Twist1/2. They can act as direct or indirect repressors of E-cadherin..$^{91,92}$ The expression of these EMT factors is regulated through different signaling systems (i.e., RTKs, Notch, and WNT signaling), and is often associated with poor prognosis and high probability of tumor recurrence. ${ }^{93}$ The other hallmarks of EMT are loss of tight junction proteins (i.e., coxsackie and adenovirus receptor-CXADR, occludin) and high probability of destroyment of epithelial barrier. ${ }^{93,94}$ The enhanced migratory properties of EMT cells is associated with increased expression of mesenchymal proteins (i.e., intermediate filament protein vimentin). ${ }^{91}$ Other properties of EMT cells associated with cancer progression and metastasis are linked with the possibility to avoid senescence. As a result of inhibition of tumor suppressor proteins p16 and p21 the cellular senescence mechanisms are downregulated and EMT is induced. ${ }^{95}$ Furthermore, the role of EMT in drug resistance has been reported. ${ }^{84-96}$ In breast and pancreatic cancer cases has been revealed, that drug resistance is accompanied with EMT. ${ }^{85-97}$ High levels of E-cadherin are predictive factor for sensitivity to epidermal growth factor receptor (EGFR) kinase inhibitors. Cells expressing EMT markers have also been shown to have a higher resistance to oxaliplatin and paclitaxel. ${ }^{98,99}$ Recent studies highlight the role of EMT as an important mechanism of chemoresistance. ${ }^{100-102}$

\section{Epigenetic aspects}

Cancer related immune processes and mechanisms are complex. They entail the modifications in cancer cells and tumor microenvironment. These alterations aim inhibition of tumor cells recognition by immune competent cells. They include modified expression and presentation of tumor-associated antigens (TAAs) and secretion of cytokines. These complex processes are driven by different factors including altered epigenetic marks in tumor cells. It has been recently revealed, that epigenetic modifications can alter immune cell phenotype as well as function. ${ }^{103}$ Both regulatory and cytolytic functions of the immune cell may be altered. The accumulation of tumorigenesis specific epigenetic alterations contributes to obvious changes in genome-wide transcriptional regulation and genetic stability those are the molecular background of immune evasion. As it is mentioned above, epigenetic aspects of tumorigenesis are important and essential for suppression of immune recognition and immune surveillance. They promote immune evasion through alterations of tumor and microenvironment. Immunosuppression is a striking feature of the global methylation pattern of the cancer genome. It is common and extends across heterogenous cancer phenotypes. The potential for epigenetic modifying agents (EMAs) to reverse these phenomena is an excellent therapeutic approach. The potential of epigenetic therapies before or in combination with immune therapies have been recently reported. These can be achieved through a variety of mechanisms enhancing antitumor immune responses. For example, these mechanisms may include improvement of immune recognition via expression, processing, and presentation of TAAs in tumor cells and efficient recognition, T-cell activation, and lysis of tumor targets by immune cells. Epigenetic therapies could play different roles within this complexity of an effective antitumor immune response. ${ }^{103}$ other aspects of cancer related epigenetics is the methylation of tumor-suppressor genes and some miRNAs encoding genes. They can be used as important predictive and prognostic tools of cancer. Such approach acquires the especial importance during application of HDAC inhibitors for target gene expression activation. ${ }^{104}$ In breast cancer the methylation of RASSF1A, HOXA5, TWIST1, CCND2, p16, BRCA1, as well as genes encoding the estrogen receptor (ESR1) and the progesterone receptor (PGR) has been reported. These genes are associated with cancer genesis. ${ }^{105}$ Epigenetic changes specific to other tumor types may also provide prognostic data in ovarian cancer, ${ }^{106}$ prostate cancer, ${ }^{107}$ glioblastoma and cutaneous tumors. ${ }^{108}$

\section{Conclusion}

Immune reactions during cancer as well as the underlying this phenomenon, molecular mechanisms of cancer cells and immuno competent cells are important and actual research topics. The special research emphasis is given to the cellular communications and tumor tissue microenvironment. The immunology response against cancer cells can be determined as the complex task, which can be successfully performed only in the conditions of the adjusted and coordinated intracellular mechanisms, cell-cell and cell-matrix interactions. The target of modern research is focused on determination of genes critical for immune escape and metastasis as well as on their regulation. The data of this research will ensure the development of the modern and effective anti-cancer therapies as well as successful realization of the existed ones. 


\section{Acknowledgements}

The authors thank New Vision University for providing access to the publication resources.

\section{Conflict of interest}

The authors declare there is no conflict of interest.

\section{References}

1. Aptsiauri N, Cabrera T, Mendez R, et al. Role of altered expression of HLA class I molecules in cancer progression. In: Shurin MR, Smolkin YS editors Immune-Mediated Diseases. Adv Exp Med Biol. 2007;601:123-131.

2. Shenoy SK, Drake MT, Nelson ChD, et al. $\beta$-Arrestin-dependent, G protein-independent ERK1/2 activation by the 2 adrenergic Receptor. The Journ of Biol Chem. 2006;281(2):1261-1273.

3. Boon T, Coulie PG, Van den Eynde BJ, et al. Human T cell responses against melanoma. Annu Rev Immunol. 2006;24:175-208.

4. Van Raamsdonk C, Griewank K, Michelle B Crosby MB, et al. Mutations in GNA11 in Uveal Melanoma. $N$ Engl $J$ Med. 2010;363:2191-2199.

5. Remeseiro S, Cuadrado A, Carretero M, et al. Cohesin-SA1 deficiency drives aneuploidy and tumourigenesis in mice due to impaired replication of telomeres. EMBO J. 2012;31(9):2076-2089.

6. Cheng AL, Kang YK, Chen Z, et al. Efficacy and safety of sorafenib in patients in the Asia-Pacific region with advanced hepatocellular carcinoma: a phase III randomised, double-blind, placebo-controlled trial. Lancet Oncol. 2009;10(1):25-34.

7. Paschen A, Arens N, Sucker A, et al. The coincidence of chromosome 15 Aberrations and $\beta 2$-microglobulin gene mutations is causative for the total loss of human leukocyte antigen class I expression in melanoma. Clin Cancer Res. 2006;12(11 pt 1):3297-305.

8. Gembarska A, Luciani F, Fedele C, et al. MDM4 is a key therapeutic target in cutaneous melanoma. Nat Med. 2012;18(8):1239-1247.

9. Iwatsuki M, Mimori K, Yokobori T, et al. Epithelia-mesenchymal transition in cancer development and its clinical significance. Cancer Sci. 2010;101(2):293-299.

10. Kokkola R, Andersson A, Mullins G, et al. RAGE is the major receptor for the proinflammatory activity of HMGB1 in rodent macrophages. Scand J Immunol. 2005;61(1):1-9.

11. Parry RV, Chemnitz JM, Frauwirth KA, et al. CTLA-4 and PD-1 receptors inhibit T-Cell activation by distinct mechanisms. Mol Cell Biol. 2005; 25(21):9543-53.

12. Iwai Y, Ishida M, Tanaka Y, et al. Involvement of PD-L1 on tumor cells in the escape from host immune system and tumor immunotherapy by PD-L1 blockade. Proc Natl Acad Sci USA. 2002;99(19):12293-12297.

13. Mani A, Guo W, Liao MJ, et al. The epithelial-mesenchymal transition generates cells with properties of stem cells. Cell. 2008;133(4):704 715 .

14. Azuma T, Yao S, Zhu G, et al. B7-H1 is a ubiquitous antiapoptotic receptor on cancer cells. Blood. 2008;111(7):3635-3643.

15. Gradishar W, Anderson B, Blair S, et al. Breast cancer version 3.2014 J Natl Compr Canc Netw. 2014;12(4):542-590.
16. Schalper K, Velcheti V, Carvajal D, et al. In situ tumor PD-L1 mRNA expression is associated with increased TILs and better Outcome in. Breast Carcinomas. Clin Cancer Res. 2014;20(10):2773-2782.

17. Ghebeh H, Barhoush E, Tulbah A, et al. FOXP3+ Tregs and B7$\mathrm{H} 1+$ /PD-1+T lymphocytes co-infiltrate the tumor tissues of high-risk breast cancer patients: Implication for immunotherapy. BMC Cancer. 2008;8:57.

18. Muenst S, Schaerli AR, Gao F, et al. Expression of programmed death ligand 1 (PD-L1) is associated with poor prognosis in human breast cancer. Breast Cancer Res Treat. 2014;146(1):15-24.

19. Twyman-Saint VC, Rech AJ, Maity A, et al. Radiation and dual checkpoint blockade activate non-redundant immune mechanisms in cancer. Nature. 2015;520(7547):373-377.

20. Taube JM, Klein A, Brahmer JR, et al. Association of PD-1, PD-1 ligands, and other features of the tumor immune microenvironment with response to Anti-PD-1 therapy. Clin Cancer Res. 2014;20(19):5064-74.

21. Gubin MM, Zhang X, Schuster H, et al. Checkpoint blockade cancer immunotherapy targets tumour-specific mutant antigens. Nature. 2014;515(7528):577-81

22. Snyder A, et al. Genetic basis for clinical response to CTLA-4 blockade in melanoma. N Engl J Med. 2014;371:2189-2199.

23. Rizvi NA, Hellmann MD, Snyder A, et al. Cancer immunology. Mutational landscape determines sensitivity to PD-1 blockade in nonsmall cell lung cancer. Science. 2015;348(6230):124-128.

24. Le DT, Uram JN, Wang H, et al. PD-1 Blockade in tumors with mismatch-repair deficiency. $N$ Engl J Med. 2015;372(26):2509-2520.

25. Ma W, Gilligan BM, Yuan J, et al. Current status and perspectives in translational biomarker research for PD-1/PD-L1 immune checkpoint blockade therapy. J Hematol Oncol. 2016;9:47.

26. Festino L, Botti G, Lorigan P, et al. Cancer treatment with anti-pd-1/ pd-11 agents: is pd-11 expression a biomarker for patient selection? Drugs. 2016;76(9):925-945.

27. Muro K, Chung HC, Shankaran V, et al. Pembrolizumab for patients with PD-L1-positive advanced gastric cancer (KEYNOTE-012): a multicentre, open-label, phase 1b trial. Lancet Oncol. 2016;17:717726.

28. Ribas A, Caroline Robert, F. Stephen Hodi, et al. Association of response to programmed death receptor 1 (PD-1) blockade with pembrolizumab (MK-3475) with an interferon-inflammatory immune gene signature. 2015 ASCO Annual Meeting. J Clin Oncol. 2015;33 suppl 15:3001.

29. Mandai M, Hamanishi J, Abiko K, et al. Anti-PD-L1/PD-1 immune therapies in ovarian cancer: basic mechanism and future clinical application. Int J Clin Oncol. 2016;21(3):456-461.

30. Sunshine J, Taube JM. Pd-1/Pd-L1 Inhibitors. Curr Opin Pharmacol. 2015;23:32-38.

31. Disis ML, Manish R Patel, Shubham Pant, et al. Avelumab (MSB0010718C; anti-PD-L1) in patients with recurrent/refractory ovarian cancer from the JAVELIN Solid Tumor phase Ib trial: Safety and clinical activity. ASCO Annual Meeting. J Clin Oncol. 2015;34 suppl 15:5533.

32. Hugo W, Zaretsky JM, Sun L, et al. Genomic and transcriptomic features of response to anti-pd-1 therapy in metastatic melanoma. Cell. 2016;165(1):35-44. 
33. Imamoto A, Soriano P, Stein PL. Genetics of signal transduction: tales from the mouse. Curr Opin Genet Dev. 1994;4(1):40-46.

34. Hunter T. Signaling--2000 and beyond. Cell. 2000;100(1):113-127.

35. Pawson T. Specificity in signal transduction: from phosphotyrosineSH2 domain interactions to complex cellular systems. Cell. 2004;116(2):191-203

36. Vogelstein B, Kinzler KW. Cancer genes and the pathways they control. Nat Med. 2004;10(8):789-799.

37. Hanahan D, Weinberg RA. The hallmarks of cancer. Cell. 2000;100(1):57-70

38. Lemmon MA, Schlessinger J. Cell signaling by receptor tyrosine kinases. Cell. 2010; 141(7):1117-1134.

39. Chin L, Andersen JN, Futreal PA. Cancer genomics: from discovery science to personalized medicine. Nat Med. 2011;17(3):297-303.

40. Rubin BP, Heinrich MC, Corless CL. Gastrointestinal stromal tumour. Lancet. 2007; 369(9574):1731-1741.

41. Garnett MJ, Marais R. Guilty as charged: B-RAF is a human oncogene Cancer Cell. 2004;6(4):313-319.

42. Harris TJ, McCormick F. The molecular pathology of cancer. Nat Rev Clin Oncol. 2010;7(5):251-265.

43. Luo J, Manning BD, Cantley LC. Targeting the PI3K-Akt pathway in human cancer: rationale and promise. Cancer Cell. 2003;4(4):257262.

44. Di Cristofano A, Pandolfi PP. The multiple roles of PTEN in tumor suppression. Cell. 2000;100(4):387-390.

45. Chin L, Andersen JN, Futreal PA. Cancer genomics: from discovery science to personalized medicine. Nat Med. 2011;17(3):297-303.

46. Gumbiner BM. Cell adhesion: the molecular basis of tissue architecture and morphogenesis. Cell. 1996;84:345-357.

47. Giancotti FG, Ruoslahti E. Integrin signaling. Science. 1999;285(5430):1028-1032.

48. Pylayeva Y, Gillen KM, Gerald W, et al. Ras-and PI3K dependent breast tumorigenesis in mice and humans requires focal adhesion kinase signaling. J Clin Invest. 2009;119(2):252-266.

49. Bolen JB, Veillette A, Schwartz AM, et al. Activation of pp60c-src protein kinase activity in human colon carcinoma. Proc Natl Acad Sci USA. 1987;84(8):2251-2255.

50. Clevers H. Wnt/beta-catenin signaling in development and disease Cell. 2006;127(3):469-480.

51. Okada T, You L, Giancotti FG. Shedding light on Merlin's wizardry. Trends Cell Biol. 2007;17(5):222-229.

52. Cavallaro U, Christofori G. Cell adhesion and signalling by cadherins and Ig-CAMs in cancer. Nat Rev Cancer. 2004;4(2):118-132.

53. Cooke NM, Spillane CD, Sheils O, et al. Aspirin and P2Y12 inhibition attenuate platelet-induced ovarian cancer cell invasion. BMC Cancer. 2015;15:627.

54. Becker JC, Andersen MH, Schrama D, et al. Immune-suppressive properties of the tumor microenvironment. Cancer Immunol Immunother. 2013;62(7):1137-1148.

55. Zamarron BF, Chen W. Dual roles of immune cells and their factors in cancer development and progression. Int J Biol Sci. 2011;7(5):651658.
56. Blackwill F, Mantovani A. Inflammation and cancer: back to virchow? Lancet. 2002;357(9255):539-545.

57. Rakoff-Nahoum S. Why cancer and inflammation. Yale J Biol Med. 2007;79(3-4):123-130.

58. Grivennikov SI, Greten FR, Karin M. Immunity, inflammation, and cancer. Cell. 2010;140(6):883-899.

59. de Martel C, Franceschi S. Infections and cancer: established associations and new hypotheses. Crit Rev Oncol Hematol. 2009;70(3):183-194.

60. Coussens LM, Werb Z. Inflammatory cells and cancer: think different! J Exp Med. 2001;193(6):F23-26.

61. Coussens LM, Werb Z. Inflammation and cancer. Nature. 2002;420(6917):860-867.

62. Dunn GP, Bruce AT, Ikeda H, et al. Cancer immunoediting: from immunosurveillance to tumor escape. Nat Immunol. 2002;3(11):991998.

63. Dunn GP, Old LJ, Schreiber RD. The three Es of cancer immunoediting Annu Rev Immunol. 2004;22:329-360.

64. Sawnn JB, Smyth MJ. Immune surveillance of tumors. J Clin Investig. 2007;117(5):1137-1146

65. Shay JW, Roninson IB. Hallmarks of senescence in carcinogenesis and cancer therapy. Oncogene. 2004;23(16):2919-2933.

66. Chow E, Danielewski JA, Fehler G, et al. Human papillomavirus in young women with Chlamydia trachomatis infection 7 years after the Australian human papillomavirus vaccination programme: a crosssectional study. Lancet Infect Dis. 2015;15(11):1314-1323.

67. Hori S, Sakaguchi S. Foxp3: a critical regulator of the development and function of regulatory T cells. Microbes and Infection. 2004;6(8):745751.

68. Ebert LM, Tan BS, Browning J, et al. The regulatory T cell-associated transcription factor foxP3 is expressed by tumor cells. Cancer Res. 2008;68(8):3001-3009.

69. Karanikas V, Speletas M, Zamanakou M, et al. FOXP3 expression in human cancer cells. J Transl Med. 2008;6:19.

70. Takenaka M, Seki N, Toh U, et al. FOXP3 expression in tumor cells and tumor-infiltrating lymphocytes is associated with breast cancer prognosis. Mol Clin Oncol. 2013;1(4):625-632.

71. Hinz S, Pagerols-Raluy L, Oberg HH, et al. FOXP3 expression in pancreatic carcinoma cells as a novel mechanism of immune evasion in cancer. Cancer Res. 2007;67(17):8344-8350.

72. Liyanage UK, Goedegebuure PS, Moore TT, et al. Increased prevalence of regulatory T cells (Treg) is induced by pancreas adenocarcinoma. $J$ Immunother. 2006;29(4):416-424

73. Li YW, Gao Q, Qiu SJ, et al. Intratumoral balance of regulatory and cytotoxic $\mathrm{T}$ cells is associated with prognosis of hepatocellular carcinoma after resection. J Clin Oncol. 2007;25(18):2586-2593.

74. Adeegbe DO, Nishikawa H. Natural and induced T regulatory cells in cancer. Front Immunol. 2013;4:190.

75. Ondondo B, Jones E, Godkin A, et al. Home sweet home: the tumor microenvironment as a heaven of regulatory T cells. Front Immunol. 2013;4:197.

76. Marrota L, Almendo V, Marusyk A, et al. The JAK2/STAT3 signaling pathway is required for growth of CD44+CD24- stem cell-like breast cancer cells in human tumors. J Clin Invest. 2011;121(7):2723-2735. 
77. Oda T, Kikkawa M. Novel structural labeling method using cryo-electron tomography and biotin-streptavidin system. JSB 2013;183(3):305-311

78. Bremnes RM, Donnem T, Al-Saad S, et al. The role of tumor stroma in cancer progression and prognosis: emphasis on carcinomaassociated fibroblasts and nonsmall cell lung cancer. $J$ Thorac Oncol. 2011;6(1):209-217.

79. Paget SFRCS. The distribution of secondary growths in cancer of the breast. Lancet. 1889;133(3421):571-573.

80. Bissell MJ, Radisky D. Putting tumours in context. Nat Rev Cancer. 2001;1(1):46-54

81. Wiseman BS, Sternlicht MD, Lund LR, et al. Site-specific inductive and inhibitory activities of MMP-2 and MMP-3 orchestrate mammary gland branching morphogenesis. J Cell Biol. 2003;162(6):1123-1133.

82. Bissell MJ, Labarge MA. Context, tissue plasticity, and cancer: are tumor stem cells also regulated by the microenvironment? Cancer Cell. 2005;7(1):17-23.

83. Lu P, Weaver VM, Werb Z. The extracellular matrix: a dynamic niche in cancer progression. J Cell Biol. 2012;196(4):395-406.

84. Spano D, Zollo M. Tumor microenvironment: a main actor in the metastasis process. Clin Exp Metastasis. 2012;29(4):381-395.

85. Hanahan D, Coussens LM. Accessories to the crime: functions of cells recruited to the tumor microenvironment. Cancer Cell. 2012;21(3):309-322.

86. Hu M, Polyak K. Molecular characterisation of the tumour microenvironment in breast cancer. Eur J Cancer. 2008;44(18):27602765 .

87. Lin $\mathrm{HJ}$, Zuo T, Lin $\mathrm{CH}$, et al. Breast cancer associated fibroblasts confer AKT1-mediated epigenetic silencing of cystatin M in epithelial cells. Cancer Res. 2008;68(24):10257-10266.

88. Kitamura T, Qian BZ, Pollard JW. Immune cell promotion of metastasis. Nat Rev Immunol. 2015;15(2):73-86.

89. Nieto MA, Huang RY, Jackson RA, et al. EMT. Cell. 2016;166(1):2145 .

90. Frixen UH, Behrens J, Sachs M, et al. E-cadherin mediated cell celladhesion prevents invasiveness of human carcinoma-cells. J Cell Biol. 1991;113(1):173-185.

91. Lamouille S, Xu J, Derynck R. Molecular mechanisms of epithelialmesenchymal transition. Nat Rev Mol Cell Biol. 2014;15(3):178-196.

92. Nieto MA, Cano A. The epithelial-mesenchymal transition under control: Global programs to regulate epithelial plasticity. Semin Cancer Biol. 2012;22(5-6):361-368.

93. Fuxe J, Vincent T, Garcia de Herreros A. Transcriptional crosstalk between tgf-beta and stem cell pathways in tumor cell invasion: Role of emt promoting smad complexes. Cell Cycle. 2012;9(12):23632374 .
94. De Craene B, Berx G. Regulatory networks defining emt during cancer initiation and progression. Nat Rev Cancer. 2013;13(2):97-110.

95. Ansieau S, Bastid J, Doreau A, et al. Induction of EMT by twist proteins as a collateral effect of tumor-promoting inactivation of premature senescence. Cancer Cell. 2008;14(1):79-89.

96. Sommers CL, Heckford SE, Skerker JM, et al. Loss of epithelia markers and acquisition of vimentin expression in adriamycinresistant and vinblastine resistant human breast-cancer cell-lines. Can Res. 1992;52(19):5190-5197.

97. Singh A, Settleman J. Emt, cancer stem cells and drug resistance: an emerging axis of evil in the war on cancer. Oncogene. 2010;29(34):4741-4751.

98. Kajiyama H, Shibata K, Terauchi M, et al. Chemoresistance to paclitaxel induces epithelial-mesenchymal transition and enhances metastatic potential for epithelial ovarian carcinoma cells. Int J Oncol. 2007;31(2):277-283.

99. Yang AD, Fan F, Camp ER, et al. Chronic oxaliplatin resistance induces epithelial-to-mesenchymal transition in colorectal cancer cell lines. Clin Cancer Res. 2006;12(14 pt 1):4147-4153.

100. Fischer KR, Durrans A, Lee S, et al. Epithelial-to-mesenchymal transition is not required for lung metastasis but contributes to chemoresistance. Nature. 2015;527(7579):472-476.

101. Shibue T, Weinberg RA. Emt, cscs, and drug resistance: The mechanistic link and clinical implications. Nat Rev Clin Oncol. 2017;14(10):611-629.

102. Zheng X, Carstens JL, Kim J, et al. Epithelial-to-mesenchymal transition is dispensable for metastasis but induces chemoresistance in pancreatic cancer. Nature. 2015;527(7579):525-530.

103. Heninger E, Kruger TEG, Lang JM. Augmenting antitumor immune responses with epigenetic modifying agents. Frontiers in Immunol. 2015;6:29.

104. Kobayashi N, Hiraoka N, Yamagami W, et al. FOXP3+ regulatory T cells affect the development and progression of hepatocarcinogenesis. CCR. 2007;3(3):902-911.

105. Dworkin AM, Spearman A, Tseng S, et al. Methylation not a frequent "second hit" in tumours with germline BRCA mutations. Fam Cancer. 2009;8(4):339-346.

106. Thiery JP, Acloque H, Huang R, et al. Epithelial-mesenchymal transitions in development and disease. Cell. 2009;39(5):871-890.

107. Bastian P, Yegnasubramanian S, Palapattu G, et al. Molecular biomarker in prostate cancer: The role of $\mathrm{CpG}$ island hypermethylation. Eur Urol. 2004;46(6):698-708.

108. Nagarajan R, Costello J. Molecular epigenetics and genetics in neurooncology. Neurotherapeutics. 2009;6(3):436-446. 